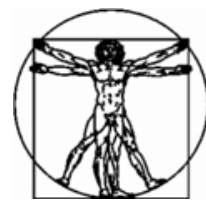

\title{
ПОСТРОЕНИЕ ЧЕТЫРЕХКАМЕРНОГО ГЕОМЕТРИЧЕСКОГО ОБРАЗА СЕРДЦА ЧЕЛОВЕКА НА ОСНОВЕ РЕНТГЕНОВСКОЙ ТОМОГРАФИИ
}

\author{
И.Н. Шардаков, А.П. Шестаков \\ Институт механики сплошных сред Уральского отделения Российской академии наук, Россия, 614013, \\ Пермь, ул. Академика Королева, 1, e-mail: shardacov@icmm.ru, shap@icmm.ru
}

\begin{abstract}
Аннотация. Моделирование отдельных органов человека и организма в целом открывает дополнительные возможности в выявлении патологий, назначении оптимальных медицинских воздействий и прогнозировании их последствий. Для реализации этого подхода необходим геометрический образ органа. Преимущественно он представляет собой конечно-элементную сетку, поскольку реализация моделей, описывающих физико-механические процессы, протекающие в органе, осуществляется численно и в большинстве случаев методом конечных элементов. В настоящей работе рассматривается построение конечно-элементного образа сердца человека. Процесс построения основан на результатах мультисрезовой рентгеновской томографии, полученных in vivo. Исходная томограмма обрабатывается специально разработанным морфологическим фильтром, который позволяет сглаживать границу органа, устранять шумовой сигнал томографа и исключать элементы органа заданного масштаба. С помощью этого алгоритма выполнена фильтрация внешней границы сердца и его полостей. На основе обработанного томографического образа с помощью алгоритма роста создан трехмерный четырехкамерный геометрический образ сердца. Для этого образа построена граница в виде поверхностной сетки и проведены ее обработка с целью устранения дефектов в виде самокасаний, а также сглаживание, которое позволило устранить ломаный характер поверхности. Эти поверхности экспортированы в блок генерации сеток ANSYS ICEM CFD, в котором осуществлено построение конечно-элементного аналога сердца человека. Разработанный алгоритм обладает высокой универсальностью и может быть использован для построения геометрических образов других органов человека, единственным ограничением является обеспечение достаточного контраста томографического сигнала между восстанавливаемым органом и окружающей средой.
\end{abstract}

Ключевые слова: сердце, сегментация, морфологическая фрильтрация, томограмма, конечные элементы.

\section{ВВЕДЕНИЕ}

Моделирование отдельных органов человека и организма в целом открывает дополнительные возможности в выявлении патологий, назначении оптимальных медицинских воздействий и прогнозировании их последствий. Важнейшим этапом моделирования является создание геометрического образа исследуемого объекта. Решению этой задачи посвящено большое количество работ. Достаточно полно состояние исследований в данной области изложено в работе [10]. Опираясь на этот обзор, можно выделить следующие направления, связанные с построением 
геометрического образа сердца. Исторически первое направление основано на использовании анатомической информации, полученной с образца сердца in vitro путем препарирования и измерения геометрических характеристик стандартными инженерными средствами. Алгоритмы реализации такой идеологии достаточно очевидны и, как правило, позволяют учесть только общие параметры, присущие каждому человеку. Более перспективными являются неинвазивные варианты реконструкции, основанные на результатах рентгеновских, магнитоно-резонансных или ультразвуковых томографических исследований. Эти варианты позволяют учесть индивидуальные особенности конкретного человека и оценить их количественные параметры. В литературе процесс выделения объекта исследования из томографических данных называется сегментацией.

Методы сегментации томографических образов базируются на различных подходах. Первые алгоритмы были основаны на ручном построении контуров на каждом срезе и последующем автоматическом объединении их в трехмерную поверхность [22], ограничивающую моделируемый орган. К достоинству этих алгоритмов относится возможность доопределения сильно зашумленных областей сканируемого объекта на основе априорных знаний опытного врача. Эта возможность особенно актуальна при плохом качестве томограммы. К недостаткам данного направления относятся: зависимость качества геометрического образа от опыта врача, большие временные затраты и, как следствие, невозможность применения в повседневной медицинской практике. Следующий подход называется методом деформируемых поверхностей [17], согласно ему строится итерационная процедура, на каждом шаге которой поверхность деформируется так, чтобы доставлять минимум функционалу, зависящему от первых и вторых производных поверхности и внешнего поля. Преимуществами данного метода являются: возможность построения автоматического алгоритма, получение сглаженной поверхности, ограничивающей тело, возможность идентификации прерывистой границы. Однако при применении данного метода стартовая поверхность должна быть достаточно близка к истинной, и для идентификации многосвязных областей необходимо рассматривать дополнительные алгоритмы. Более универсальным подходом является метод фронтального роста [5], согласно которому исследуемая область представляется как совокупность подобластей. Этот метод является также итерационным, на каждой итерации к текущей области роста добавляются соседние подобласти, удовлетворяющие заданному критерию подобия. Процесс выполняется до тех пор, пока количество элементов, добавленных за итерацию, не станет равным нулю. Метод позволяет построить автоматический алгоритм, результатом которого является замкнутая область. Кроме этого, метод обладает высокой скоростью и применим для односвязных и многосвязных восстанавливаемых областей.

В настоящее время большое распространение приобретают статистические модели формы. Они позволяют учитывать априорные знания о моделируемом органе и поэтому делают процесс реконструкции устойчивым к шумам и артефактам. Первоначально в этих методах строится множество базисных форм, которые являются геометрическими представлениями конкретных органов. Затем геометрия реконструируемого органа представляется в виде линейной комбинации базисных форм. Коэффициенты этой комбинации выбираются так, чтобы минимизировать отклонение получаемой геометрии от органа, представленного в томографическом образе. Разные варианты этого подхода описаны в обзорной статье $[11,19]$. Основываясь на данном подходе, в работе [7] описан процесс создания трехмерного образа сердца. Необходимо отметить, что для данного подхода существует вероятность нехватки базисных форм для описания индивидуальных особенностей реконструируемого органа. 
В настоящей работе использован алгоритм роста, авторская реализация которого изложена в работе [3]. Данный метод универсален и позволяет реконструировать объекты любой сложности. Однако при реконструкции сердца человека возникают проблемы, связанные с зашумлённостью томографического сигнала и устранением из его структуры большого количества трабекулярных и папиллярных мышц. Учет этих мышц приводит к существенному увеличению вычислительных затрат, не связанных с получением новых качественных и количественных закономерностей моделируемых процессов. В частности, для задач электродинамики сердца было показано [4], что учет этих особенностей не приводит к значительным изменениям результатов. Поэтому в настоящей работе выполняется построение геометрического образа, отражающего первый масштабный уровень, который определяет расположение стенок сердца в пространстве и их толщины. Для преодоления описанных проблем разработан алгоритм фильтрации, который можно отнести к классу морфологических фильтров [14]. Однако здесь вместо стандартных алгоритмов эрозии и расширения используются их аналоги, основанные на концентрации тела в области фильтра. Описание и применение этого алгоритма изложены в следующем разделе.

Кроме развития методов сегментации, много усилий было приложено, чтобы улучшить методы их реализации. Это вызвано увеличением количества данных, содержащихся в томограммах и в результате увеличения времени, требуемого для их сегментации. Одним из приближений для увеличения скорости сегментации является применение метода параллельных вычислений при нахождении единиц графики. Значительное количество таких методов описано в обзорной статье.

Построение геометрического образа как основы для моделирования можно считать завершенным, если сформирована вся информация, необходимая для решения задачи математической физики современными численными методами, среди которых одно из ведущих мест занимает метод конечных элементов. Для реализации этого метода необходимо решать достаточно сложную алгоритмическую задачу, связанную с дискретизацией расчетной области на конечные элементы. В настоящей работе эта задача решена средствами модуля генерации сеток ANSYS ICEM CFD.

При реконструкции сердца в настоящей работе использованы материалы мультисрезовой рентгеновской томографии, представленные на сайте [6]. Полное название образа: Alias Name: TOUTATIX; Modality: CT; File Size: 195 MB; Description: SCoronary artery anomaly (emerging from pulmonary artery). Пространственное разрешение: в томографическом слое, мм, между слоями - 0,5 мм.

\section{АЛГОРИТМ ФИЛЬТРАЦИИ}

Предлагаемый алгоритм является обобщением известных методов морфологической фильтрации [14] - расширения (рис. 1,a) и эрозии (рис. 1,б). В отличие от последних, в предлагаемом методе вводится параметр $k$, называемый пороговой концентрацией. Этот параметр определяет поведение фильтра. В частности, при $k=0$ результат фильтрации тождественен процедуре «расширения» (увеличение границы обрабатываемого объекта), а при $k=1$ аналогичен процедуре «эрозии» (уменьшение границы). Положение элемента фильтра при различных параметрах концентрации изображено на рисунке (рис. 1, ).

Для сглаживания геометрических объектов используют последовательное применение эрозии и расширения. Если сначала применить эрозию и после этого расширение, то выпуклые вершины фигуры будут сглажены, но вогнутые вершины останутся без изменений (рис. 2,a). Такой фильтр имеет название «открытие». 
В случае, когда сначала применяется дилатация, а потом эрозия, происходит сглаживание вогнутых вершин, как показано на рис. 2, б. Такой фильтр называется «закрытие». Для того чтобы получить сглаживание границы в предлагаемом подходе, необходимо коэффициент концентрации установить равным 0,5 , что позволит сгладить вогнутые и выпуклые вершины одновременно. Результат фильтрации приведен на рис. 3. Следует отметить, что временные затраты этого подхода в четыре раза меньше, чем при использовании стандартных морфологических операций, так как для получения сглаживания всех вершин объекта необходимо выполнить фильтры «открытие» и «закрытие», что представляет собой четыре базовых операции «расширение» и «эрозия».

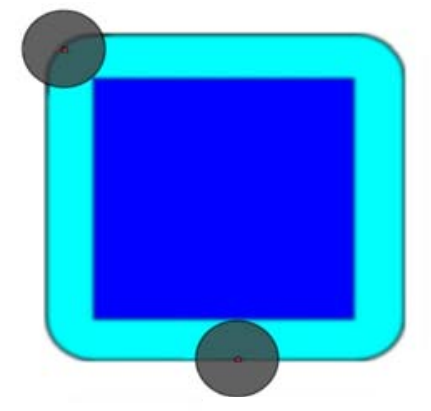

$a$

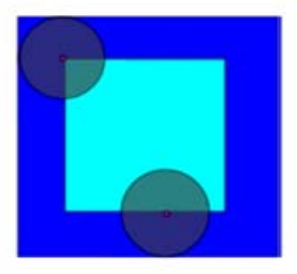

$\sigma$

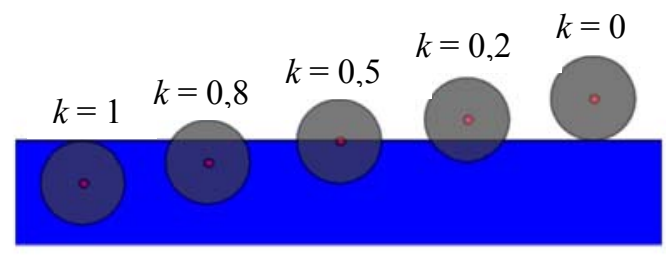

B

Рис. 1. Базовые морфологические операции: $a$ - «расширение», светлым показана область, добавленная к исходной; $\sigma$ - «эрозия», светлым показан результат фильтрации; в - положение элемента фильтра относительно границы при различных параметрах концентрации
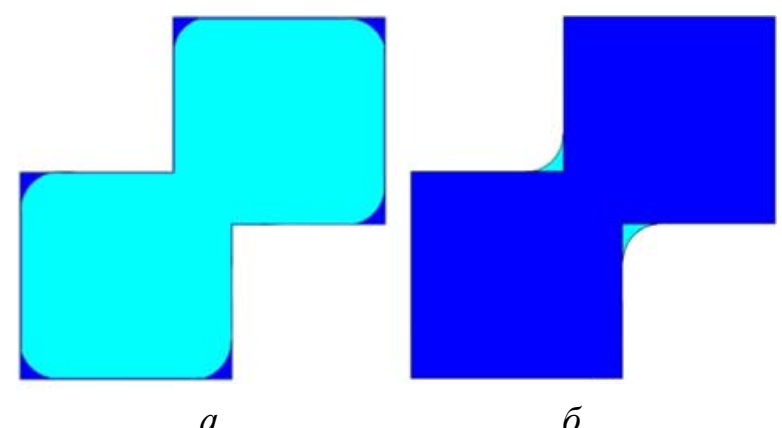

$\sigma$

Рис. 2. Стандартные морфологические фильтры сглаживания границы: $a-$ «открытие», светлым показан результат фильтрации; $\sigma$ - «закрытие», светлым показана область, добавленная к исходной

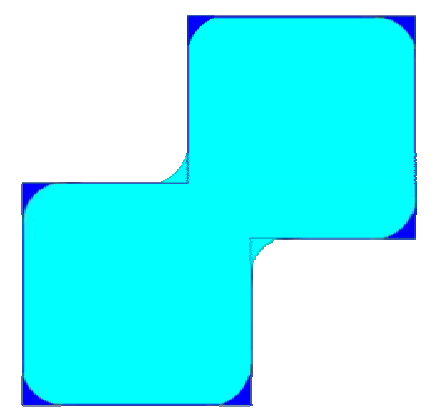

Рис. 3. Обобщенный морфологический фильтр. Коэффициент концентрации составляет 0,5. Светлым показан результат фильтрации 


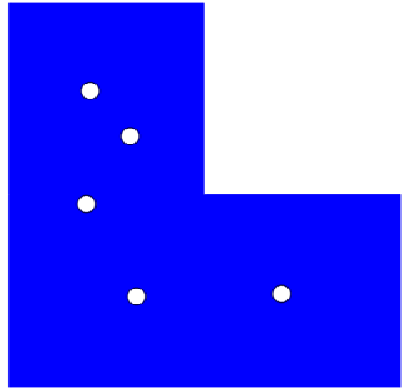

$a$

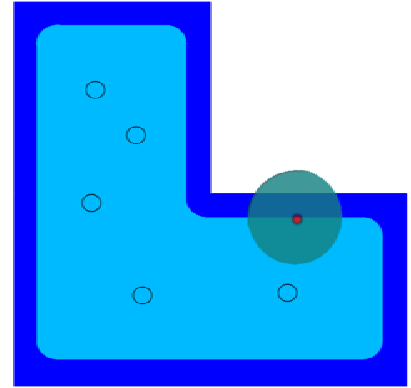

6

Рис. 4 Фильтрация внутренних элементов области: $a$ - исходная область с внутренними элементами; $\sigma$ - отфильтрованная область (внутренние элементы отсутствуют, светлым показана область, измененная фильтром)

Еще одним достоинством предлагаемого алгоритма является возможность устранения внутренних элементов области (рис. 4, $a$ ), без изменения ее границы. Для этого необходимо выбрать коэффициент концентрации из диапазона $(0,5 ; 1)$ таким, чтобы при выбранном радиусе фильтра изменение границы области не происходило (рис. 4,б). При увеличении коэффициента концентрации, уменьшается влияние на границу области (см. рис. 1, в) и при некотором значении использование фильтра не вызовет изменения границы.

Радиус фильтра необходимо выбирать таким образом, чтобы отношение площадей максимального из внутренних элементов области к площади фильтра было меньше величины $(1-k)$, это обеспечит заполнение внутренних элементов области. В результате применения фильтра с такими параметрами внутренние элементы области будут устранены, а ее граница останется неизменной. Это позволяет устранить папиллярные мышцы, располагающиеся в желудочках сердца.

\section{Описание алгоритма}

Описание алгоритма включает следующие стандартные обозначения [1]. Исходная сеточная функция $I(\bar{z})$, определенная на пространстве рациональных чисел $Z^{n}$, где $n$ - размерность пространства. Структурный элемент фильтра, представляющий собой множество

$$
B^{r}=\left\{\vec{z} \in Z^{n} \mid \forall i, z_{i} \in[-r, r]\right\}, r \in N, i \in[1, n],
$$

где $r$ - радиус фильтра; $N$ - множество целых чисел; $z_{i}$ - компонента вектора с индексом $i$. Смещенное на вектор $s$ структурное множество:

$$
B_{s}^{r}=\left\{\vec{b}+\vec{s} \mid \vec{b} \in B^{r}\right\}, \forall \vec{s} \in Z^{n} .
$$

Специальные величины, необходимые для описания алгоритма, приведены ниже. Диапазон интенсивности, соответствующий фильтруемому телу, имеет вид $\left[I_{\min }, I_{\max }\right]$. Множество подобластей из $B_{s}^{r}$, принадлежащих заданному диапазону:

$$
Q_{s}^{r}=\left\{q \in B_{s}^{r} \mid I(q) \in\left[I_{\min }, I_{\max }\right]\right\} .
$$

Множество подобластей, в окрестности которых (радиусом $r$ ) концентрация тела больше пороговой $k$, определяется соотношением 


$$
R_{k}^{r}=\left\{s \in Z^{n} \mid \frac{\left|Q_{s}^{r}\right|}{\left|B_{s}^{r}\right|}>k\right\},
$$

где параметр $k$ - пороговая концентрация. Используя введенные обозначения, процедура преобразования образа $I(z)$ принимает вид

$$
I(\vec{z})=\frac{I_{\min }+I_{\max }}{2}, \vec{z} \in R_{k}^{r}
$$

\section{Применение алгоритма}

При $k \in[0 ; 0,5)$ фильтр обладает свойством эрозии с показателем $k$, а при $k \in(0,5 ; 1]$ свойством расширения с показателем $k$. Проиллюстрируем алгоритм на примере фильтрации левого желудочка сердца человека (рис. 5).

В результате фильтрации мелкие структуры, соответствующие желудочку, устранены и получена область с гладкой границей. Величиной максимальной кривизны границы можно управлять с помощью радиуса фильтра $r$ при $k=0,5$. Для того чтобы устранить крупные структуры желудочка, такие как папиллярные мышцы, не изменяя при этом его границы, следует установить пороговую концентрацию $k>0,5$. Это позволяет обеспечить фиксированный отступ от границы и радиус фильтра $r$ достаточный для устранения выбранного объекта. Для того, чтобы устранить шумы обусловленные томографом; следует использовать $k=0,5$ и $r=1$. Такой подход устранит точечные выбросы на томографическом образе без размытия границы.

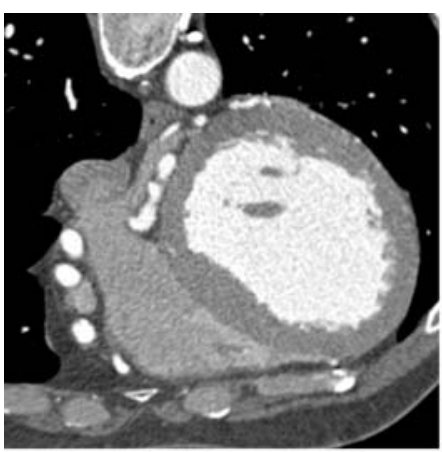

$a$

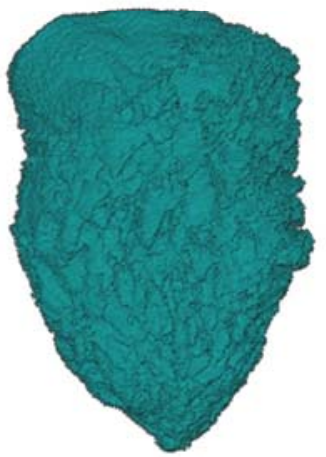

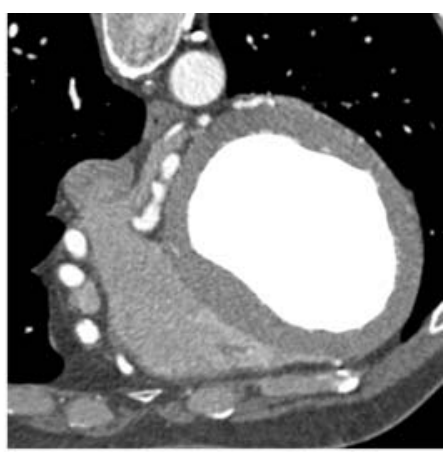

$\sigma$

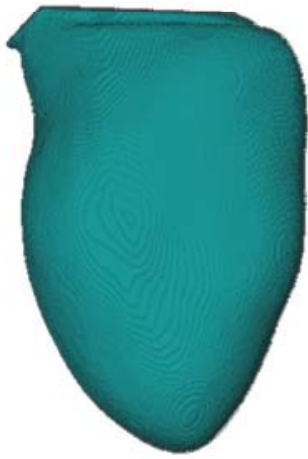

2

Рис. 5. Фильтрация левого желудочка сердца человека: до фильтрации сечение (a) и 3D-образ (в); после фильтрации сечение (б) и 3D-образ (2) 


\section{Оптимизация алгоритма}

Реализация предложенного метода фильтрации требует вычисления количества точек сеточной функции, принадлежащих заданному диапазону, в пределах структурного элемента фильтра. Эта операция занимает значительное время, поэтому алгоритм, реализующий данную функцию, должен быть оптимальным с точки зрения времени выполнения. Несмотря на высокую производительность современных компьютеров, выбор этого алгоритма является актуальной задачей, поскольку трехмерные томографические образы содержат большое количество данных. В данном исслдовании обрабатывается образ с размерами $512 \times 512 \times 308$ элементов, их общее количество - 80,7 млн. В данном случае образ можно трактовать как массив данных. Простая операция вычисления суммы всех элементов массива на компьютере с процессором Intel Core I7 на одном процессоре занимает приблизительно 0,3 с. Эта величина характеризует время доступа ко всем элементам массива. В том случае, когда структурным элементом фильтра является куб и его радиус $r$ составляет 10 элементов, для обработки одной точки исходного массива необходимое количество дополнительных обращений составляет $(2 \cdot r+1)^{3}=9261$. Это значит, что среднее значение времени работы такого фильтра составит 0,3.9261=2778 с или 46 мин. Если использовать технологию параллельного программирования, то в лучшем случае время работы уменьшится в 8 раз и составит 5,7 мин, так как количество процессоров на используемой машине равно 8. Такое время работы неприемлемо в случае, когда параметры фильтра подбираются вручную. Поэтому для увеличения скорости предлагается алгоритм, идея которого изложена на примере одномерного массива $A[i]$, где $i \in[0, N]$. Целью этого алгоритма является вычисление суммы Sum $[j]$ элементов исходного массива $A[i]$ в диапазоне $i \in[j-r ; j+r]$ где $j \in[r, N-r]$. Очевидный алгоритм решения этой задачи имеет вид

$$
\operatorname{Sum}[j]=\sum_{i=j-r}^{j+r} A[i],
$$

при этом количество обращений к исходному массиву составляет $(2 \cdot r+1)$. Авторами настоящей работы предлагается более эффективный алгоритм. Он выполняется в два этапа. На первом вычисляется стартовый элемент с индексом $r$ :

$$
\operatorname{Sum}[r]=\sum_{i=0}^{2 \cdot r} A[i],
$$

а на втором вычисляются последующие элементы по правилу

$$
\operatorname{Sum}[j]=\operatorname{Sum}[j-1]-A\left[i_{1}\right]+A\left[i_{2}\right],
$$

где $i_{1}=j-r-1, \quad i_{2}=j+r$ и $j \in[r+1, N-r]$. Для вычисления суммы элементов в области фильтра требуется три обращения к исходному массиву вне зависимости от радиуса фильтра. Поэтому в одномерном случае скорость предлагаемого алгоритма (8) в $(2 \cdot r+1) / 3$ раз быстрее, чем при вычислении согласно (6). Такое отношение скоростей будем называть коэффициентом ускорения. Предложенный алгоритм легко применим к двумерному и трехмерному случаям, для которых коэффициент ускорения пропорционален $(2 \cdot r+1)^{2} / 6$ и $(2 \cdot r+1)^{3} / 9$ соответственно. При $r=10$ в объёмном случае он составляет приблизительно 1000 раз, и время одной фильтрации рассматриваемого образа принимает значение $\approx 3 \mathrm{c}$. 


\section{РЕКОНСТРУКЦИЯ СЕРДЦА ЧЕЛОВЕКА И ПОСТРОЕНИЕ ЕГО КОНЕЧНО-ЭЛЕМЕНТНОГО АНАЛОГА}

На основе разработанного алгоритма, применяя различные радиусы фильтра $r$ и пороговую концентрацию $k$, обработаны полости сердца человека (рис. 6). Для восстановления мышечной структуры сердца человека на первом этапе обработана внешняя поверхность сердца и затем из полученного образа исключены полости. Результат описанных операций представлен на рис. 7.

Одним из приложений построенного геометрического образа является использование его в расчетных пакетах программ для моделирования различных физико-механических процессов. В частности, важной задачей является моделирование насосной функции сердца. Для ее решения необходимо моделировать электромеханическую функцию миокарда совместно с динамикой жидкости. Большинство расчетных пакетов основано на методе конечных элементов, поэтому исходный геометрический образ необходимо представить в виде конечно-элементной сетки. Процесс ее построения начинается с создания поверхностей, ограничивающих геометрический образ. Создание таких поверхностей определяется структурой образа, которая представляет собой множество подобластей, в данном случае параллелепипедов. Граница этого множества состоит из наружных граней, т.е. таких, которые принадлежат только одному параллелепипеду. Такие грани имеют вид

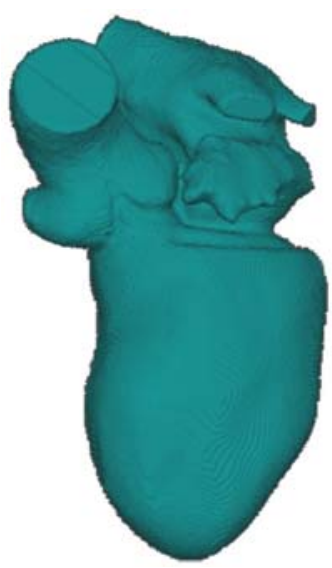

$a$

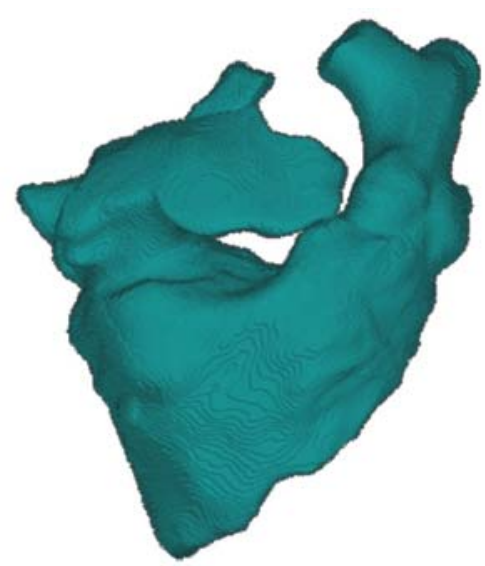

б

Рис. 6. Полости сердца человека: $a$ - левый желудочек и левое предсердие; $\sigma$ - правый желудочек и правое предсердие

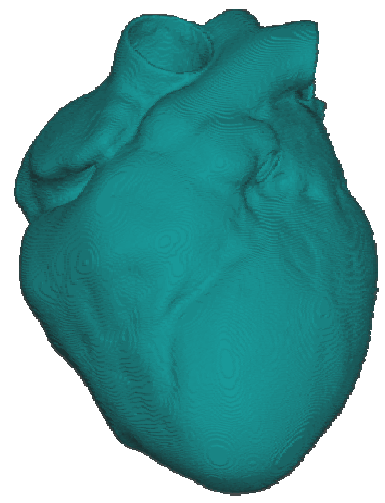

$a$

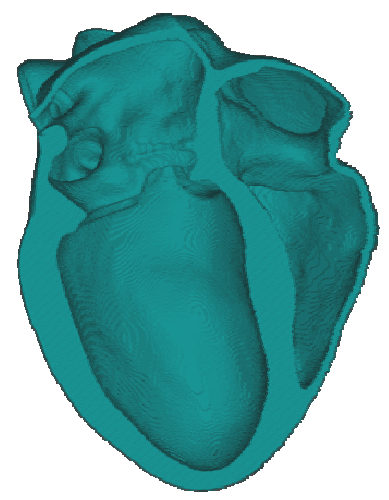

$\sigma$

Рис. 7. Мышечная структура сердца человека: $a$ - сердце целиком; $\sigma$ - сердце в разрезе 

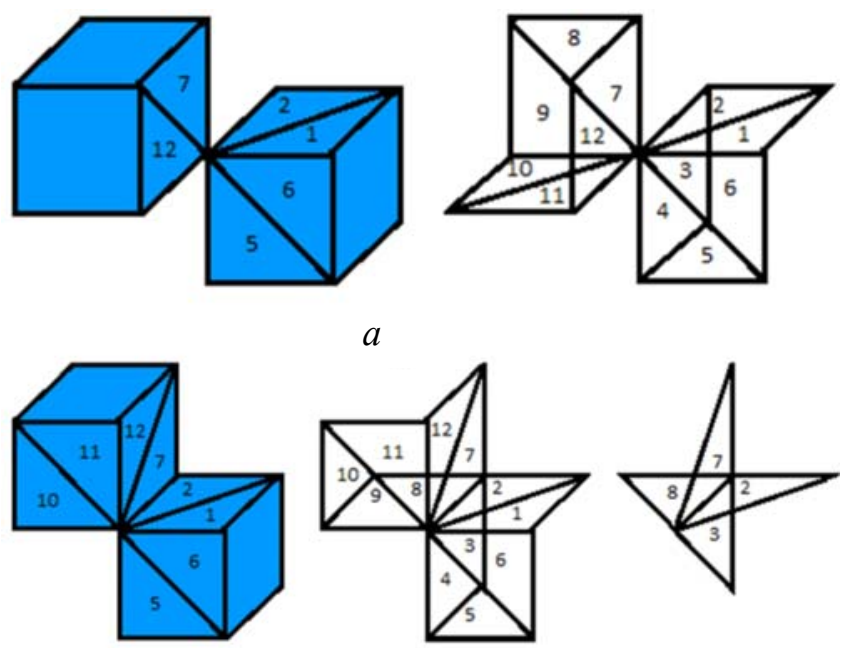

$\sigma$

Рис. 8. Иллюстрация обхода по треугольникам: $a$ - касание в узле;

б- касание по линии

четырехугольников, это не самый удобный способ описания поверхности, поэтому они разбиваются на два треугольника. В результате получается треугольная сетка, ограничивающая геометрический образ. Полученные таким образом сетки могут обладать двумя видами дефектов: самокасание в точке (рис. $8, a$ ) и по линии (рис. 8,6 ). Такие дефекты неприемлемы, поскольку они будут приводить к ошибкам при сглаживании поверхности и построении объемной сетки. Для устранения этих дефектов необходимо «расшить» узел, в котором происходит касание, т.е. создать новый узел с аналогичными координатами, но принадлежащий касающейся подобласти.

Основная идея заключается в том, что нужно обойти соседние треугольники узла так, чтобы предыдущий и последующий треугольники имели общую грань. Если после обхода остались неиспользуемые треугольники, то это значит, что в этом узле происходит касание, и необходимо создать новый узел, которым будут заменены исходные при обходе по оставшимся треугольникам. Процесс создания новых узлов повторяется до тех пор, пока количество необойденных треугольников не станет равным нулю.

Проиллюстрируем эту идею на примере устранения касаний в точке (рис. 8, a). В треугольниках с 1-го по 6-й общий узел останется без изменений, а в треугольниках с 7-го по 12-й он будет заменен на новый, с аналогичными координатами. Как следствие, поверхности, определенные треугольниками $1, \ldots, 6$ и $7, \ldots, 12$, не будут иметь общих узлов.

Для того чтобы обобщить эту идею на случай касания по линии, необходимо ввести дополнительное правило: при обходе по треугольникам следующим является тот, который имеет общую грань с предыдущим и образует с ним наименьший угол (внутренний по отношению к области роста). Рассмотрим ситуацию (рис. 8, б), когда предыдущим является треугольник 2, а последующими могут быть треугольники 7,8 , 3. Наименьшим углом обладает треугольник 3, поэтому он является истинным. В противном случае, если последующим будет выбран треугольник 7 или 8 , то это приведет к самопересечению поверхности, что невозможно для границы области, полученной с помощью алгоритма роста.

После процедуры расшивки полученную треугольную сетку необходимо сгладить, поскольку она является гранями параллелепипедов и, как следствие, является ломаной. Процедура сглаживания выполняется для всех узлов сетки и включает в себя три этапа. На первом выполняется осреднение координат узлов треугольников, 



Рис. 9. Конечно-элементный образ сердца человека: $a$ - сердце целиком, $\sigma$ - сердце в разрезе

примыкающих к сглаживаемому узлу. Затем вычисляется вектор смещения $\vec{V}=P^{m}-P^{i}$ между исходным узлом $P^{i}$ и полученным в результате осреднения $P^{m}$. В итоге, если длина вектора $|\vec{V}|$ меньше заданного максимального отклонения $\delta$, то сглаживаемому узлу $P^{r}$ присваиваются осредненные координаты $P^{r}=P^{m}$, иначе длина вектора смещения уменьшается до величины максимального отклонения, и он принимает следующее положение: $P^{r}=P^{i}+\frac{\vec{V}}{|\vec{V}|} \cdot \delta$.

Реализация процедур создания и обработки поверхностей, формирующих область сердца, выполнена в виде дополнительного модуля к программе сегментации томографических образов [3]. Из этого модуля обработанная сетка экспортируется (в формате *.STL) в сеточный генератор программного комплекса ANSYS (ANSYS ICEM $C F D)$, который формирует объемную тетраэдральную сетку с заданными параметрами разбиения (рис. 9).

В данном случае сетка, указанная на рис. 9, содержит 47474 узла и 217463 элемента. Среднее значение качества сетки равно 0,83. Этот критерий вычисляется как отношение объема элемента к радиусу вписанной сферы, нормализованному от 0 до 1.

\section{ОБСУЖДЕНИЕ}

Разработанная методика обладает высокой универсальностью и может быть использована для построения геометрических образов других органов [2]. Единственным ограничением является наличие контраста томографического сигнала между восстанавливаемым органом и окружающей средой. Используемый в этой методике алгоритм фильтрации не размывает границу восстанавливаемого объекта. Это является его главным преимуществом по сравнению с оконными фильтрами сглаживания, основанными на свертке [18]. Такое размытие при использовании алгоритма роста сильно осложняет процесс задания диапазона интенсивности, соответствующего восстанавливаемому телу. Разработанный алгоритм без дополнительных усовершенствований позволяет сглаживать объёмы произвольной топологии с удалением «незначительных» полостей и отверстий. Также можно проводить селективную фильтрацию: сначала с помощью алгоритма роста строится 
область, после этого элементы этой области помечаются и последующая фильтрация выполняется для помеченных элементов. Такой подход применяется при необходимости задания разных параметров сглаживания для разных частей органа. Оптимизированный алгоритм фильтра позволяет осуществлять одиночную фильтрацию современной томограммы высокого разрешения в течение нескольких секунд. Это делает возможным быстрый подбор параметров фильтрации для отдельных частей органа. Кроме этого, этот алгоритм достаточно прост в программной реализации, что значительно сокращает время программирования и отладки. Перечисленные достоинства алгоритма делают его хорошим выбором для предварительной обработки томограммы при использовании алгоритма роста. На завершающем этапе рассматриваемой методики осуществляется устранение некорректностей в виде самокасаний в точке и по лини. Алгоритм, реализующий эти функции, выполняет однозначную расшивку узлов, не допуская ситуации, когда в поверхности появляются «дырки» или другие дефекты сетки. Такая надежность обусловлена тем, что поверхность, образованная алгоритмом роста, является всегда замкнутой и ее самопересечение невозможно. Похожим по универсальности является метод активных поверхностей [15], для которого не требуется предварительной фильтрации. Однако этот метод обладает рядом особенностей: для областей, имеющих сложную топологию, требуются дополнительные алгоритмы, в процессе выполнения которых допускается образование новых и удаление существующих поверхностей; необходимо осуществлять контроль самопересечения поверхности; сложность задания различных параметров сглаживания для разных частей томографического образа. Перечисленные особенности усложняют алгоритм и снижают его надежность с точки зрения программной реализации. Остальные известные алгоритмы либо не позволяют выполнить автоматизацию и требуют большого количества времени при их использовании, либо применимы только для конкретного класса объектов. Таким образом, использование разработанного алгоритма фильтрации совместно с алгоритмом роста и процедурой устранения самокасаний дает надежный и эффективный способ построения трехмерного геометрического образа исследуемого объекта произвольной конфигурации. Схожая методика использована в работе [19], где сначала на основе алгоритма роста получают поверхность в виде треугольной сетки, а затем обрабатывают ее и получают трехмерную сетку.

Следует отметить, что в большинстве работ исследователи выполняют построение только левого желудочка или двух желудочков совместно $[8,16,17]$. На таких образах можно выполнять моделирование электродинамических процессов, поскольку желудочки отделены от предсердий атриовентрикулярной перегородкой, которая препятствует прохождению потенциала действия между ними. Однако при моделировании механических процессов в сердце эта перегородка не играет роли и следует учитывать взаимное влияние желудочков и предсердий. Кроме этого, моделирование насосной функции сердца, связанное с совместным решением задачи электромеханики и гемодинамики в нем, возможно только при использовании четырехкамерного образа сердца. Мышечная структура сердца анизотропна. Это имеет влияние на распространение электрических волн возбуждения и механическое сокращение сердца. В данной работе процесс ориентации волокон не рассматривается, но развитая модель может быть дополнена с учетом ориентации волокон на основе законов, полученных из аналитических данных [19], диффузной томографии [23] или рентеновских изображений высокого разрешения [13].

\section{ЗАКЛЮЧЕНИЕ}

В рамках данной работы разработана и апробирована методика, позволяющая по результатам рентгеновской томографии создавать конечно-элементные образы 
внутренних органов. В данной работе она описана на примере сердца человека. Реализация этой методики включает в себя создание алгоритма фильтрации, который позволяет устранять шумовой сигнал томографа и удалять объекты заданного масштаба. С помощью этого алгоритма выполнена фильтрация внешней границы сердца и его полостей. На основе обработанного томографического образа с помощью алгоритма роста создан трехмерный четырехкамерный геометрический образ сердца. Для этого образа построена граница в виде поверхностной сетки и проведены ее обработка с целью устранения дефектов в виде самокасаний, а также сглаживание, которое позволило устранить ломаный характер поверхности. При экспортировании этой поверхности в сеточный генератор ANSYS ICEM CFD получен конечноэлементный аналог сердца человека, который может быть использован для изучения физических процессов, реализующих насосную функцию сердца. Полученные результаты доступны для скачивания по ссылке [9] в трех форматах: база данных ANSYS, текстовое представление координат узлов и элементов, поверхность ограничивающая орган, в формате $S T L$.

\section{БЛАГОДАРНОСТИ}

Работа выполнена при финансовой поддержке РФФИ (проект № 14-01-96032).

\section{СПИСОК ЛИТЕРАТУРЫ}

1. Гонсалес Р., Вудс Р. Цифровая обработка изображений: пер. с англ. - М.: Техносфера, 2005. - 1072 с.

2. Матвеенко В.П., Шардаков И.Н., Шестаков А.П Создание конечно-элементных моделей частей скелета человека с приложениями к задаче о собственных колебаниях // Вычислительная механика сплошных сред. - 2012. - Т. 5, № 3. - С. 308-312.

3. Матвеенко В.П., Шардаков И.Н., Шестаков А.П. Алгоритм создания трехмерных образов органов человека по томографическим данным // Российский журнал биомеханики. - 2011. - Т. 15, № 4. C. $20-32$.

4. Bishop M.J., Plank G. The role of fine-scale anatomical structure in the dynamics of reentry in computational models of the rabbit ventricles // J. Physiol. - 2012. - Vol. 590. - P. 4515-4535. DOI: 10.1113/jphysiol.2012.229062.

5. Del Fresno M., Vénere M. Combined region growing and deformable model method for extraction of closed surfaces in 3D CT and MRI scans // Comput. Med. Imaging Graph. - 2009. - Vol. 33. - P. 369-376. DOI: 10.1016/j.compmedimag.2009.03.002.

6. DICOM sample image sets [Электронный pecypc]. - URL: http://www.osirix-viewer.com/datasets (дата обращения: 18.08.2015).

7. Ecabert O., Peters J. Segmentation of the heart and great vessels in CT images using a model-based adaptation framework // Med. Image Anal. - 2011. - Vol. 15. - P. 863-876. DOI: 10.1016/j.media.2011. 06.004 .

8. Einstein D.R., Kuprat A.P., Jiao X., Carson J.P., Einstein D.M., Jacob R.E., Corley R.A. An efficient algorithm for mapping imaging data to 3D unstructured grids in computational biomechanics // Int. J. Numer. Method Biomed. Eng. - 2013. - Vol. 29. - P. 1-16. DOI: 10.1002/cnm.2489.

9. FEM models [Электронный pecypc]. - URL: www.icmm.ru/tomogram-to-fem (дата обращения: 18.08.2015).

10. Frangi A.F., Niessen W.J., Viergever M.A. Three-dimensional modeling of functional analysis of cardiac images: a review // IEEE Trans Med Imaging. - 2001. - Vol. 20, No. 1. - P. 2-25.

11. Heimann T., Meinzer H. Statistical shape models for 3D medical image segmentation: a review // Med. Image Anal. - 2009. - Vol. 13. - P. 543-563. DOI: 10.1016/j.media.2009.05.004.

12. Iglesias J.E., Sabunc M.R. Multi-atlas segmentation of biomedical images: A survey. Med. Image Anal., 2015, vol. 24, no. 1, pp. 205-219. DOI: 10.1016/j.media.2015.06.012.

13. Jeffery N.S., Stephenson R.S., Gallagher J.A., Jarvis J.C., Cox P.G. Micro-computed tomography with iodine staining resolves the arrangement of muscle fibres. // J. Biomech., 2011, vol. 44, no. 1, pp. 189-192. DOI: 10.1016/j.jbiomech.2010.08.027.

14. Kim W., Kim S. 3D binary morphological operations using run-length representation // Signal Processing: Image Communication. - 2008. - Vol. 23. - P. 442-450.

15. Li B., Acton S. Active contour external force using vector field convolution for image segmentation // IEEE Trans. Med. Imaging. - 2007. - Vol. 16, № 8. - P. 38-44. 
16. Pop M., Sermesant M., Liu G., Relan J., Mansi T., Soong A., Peyrat J.M., Truong M.V., Fefer P., McVeigh E.R., Delingette H., Dick A.J., Ayache N., Wright G.A.. Construction of 3D MR image-based computer models of pathologic hearts, augmented with histology and optical fluorescence imaging to characterize action potential propagation // Med. Image Anal. - 2012. - Vol. 16. - P. 505-523. DOI: 10.1016/j.media.2011.11.007.

17. Pop M., Sermesant M., Peyrat J.-M., Crystal E., Ghate S., Mansi T., Lashevsky I., Qiang B., McVeigh E.R., Ayache N., Graham A. A 3D MRI-based cardiac computer model to study arrhythmia and its in-vivo experimental validation // FIMH. - 2011. LNCS - P. 195-205.

18. Pratt W. Digital image processing. - New York, 1978.

19. Pravdin S.F., Berdyshev V.I., Panfilov A.V., Katsnelson L.B., Solovyova O., Markhasin V.S. Mathematical model of the anatomy and fibre orientation field of the left ventricle of the heart. BioMed. Eng. OnLine, 2013, vol. 12. DOI: 10.1186/1475-925X-12-54.

20. Qing-xi Hu, Qin Xu, Yuan Yao, Qi Lu, Yuan-zhi Xu, Jiao-jiao Wang, Qi-xiang Yang. Computer aided analysis in maxillary sinus surgery // IFMBE Proceedings. - 2008. - Vol. 19. - P. 738-740.

21. Smistada E., Falcha T.L., Bozorgia M., Elstera A.C., Lindsetha F. Medical image segmentation on GPUs. A comprehensive review // Med. Image Anal. - 2015. - Vol. 20, № 1. - P. 1-18. DOI: 10.1016/j.media. 2014.10.012.

22. Treece G.M., Prager R.W. Surface interpolation from sparse cross sections using region correspondence // IEEE Trans. Med. Imaging. - 2000. - Vol. 19, № 11. - P. 23-29.

23. Vadakkumpadan F., Arevalo H., Ceritoglu C., Miller M., Trayanova N. Image-based estimation of ventricular fiber orientations for personalized modeling of cardiac electrophysiology. IEEE Trans. Med. Imaging, 2012, vol. 31, no. 5, pp. 1051-1060. DOI: 10.1109/TMI.2012.2184799.

\section{CONSTRUCTION OF THE 4-CHAMBER GEOMETRICAL IMAGE OF HUMAN HEART BASED ON X-RAY TOMOGRAPHY}

\section{I.N. Shardakov, A.P. Shestakov (Perm, Russia)}

Mathematical modelling of human organs and human body as a whole opens new possibilities for diagnosing different pathologies, prescribing an appropriate treatment and evaluating its effectiveness. A successful implementation of this problem requires generation of the geometric image of the organ. Predominantly, a finite element mesh is used since the implementation of the models describing the physical and mechanical processes occurring in the organ is done numerically and in most cases with the help of the finite element method. This paper deals with the construction of finite element image of the human heart. The process of construction is based on the results of X-ray computed tomography obtained from the in vivo studies on human heart. The initial tomographic image is processed by the specially designed morphological filter that allows us to smooth the border of the body, to eliminate noise signal of the scanner and to eliminate elements of the prescribed scale. The proposed algorithm was used to filter the external boundary of the heart and its chambers. Based on the processed tomographic images, a three-dimensional four-chamber geometric model of the human heart was constructed by applying the region growing algorithm. The boundary surface for this image is constructed in the form of a surface mesh. The subsequent processing of the mesh has been carried out to remove such defects as self-tangency. In addition, a smoothing procedure has been performed to eliminate surface roughness. These surfaces are exported to the ANSYS ICEM CFD mesh generator, which allows rapid generation of a finite element analogue of the human heart. The developed algorithm is of multi-purpose character and is equally effective in constructing geometrical models of other human organs subject to the requirement of existence of high-contrast tomographic signals, i.e. the difference in the signal strength between the reconstructed organ and environment.

Key words: human heart, segmentation, morphological filtering, tomogram, finite element model. 